\title{
Gender differences in presentation and outcome of patients with Cushing's disease in Han Chinese
}

\author{
Cheng Huan ${ }^{\mathrm{a}}$, Yuanming $\mathrm{Qu}^{\mathrm{a}}$ and Zongming Ren ${ }^{\mathrm{b}, *}$ \\ ${ }^{a}$ Shandong Provincial Hospital Affiliated to Shandong University, Ji'nan 250021, P.R. China \\ ${ }^{b}$ College of Life Science, Shandong Normal University, Ji'nan 250014, P.R. China
}

\begin{abstract}
The purpose of this study was to assess gender-related distinction in the performance of Cushing's disease (CD) regarding clinical features, radiological findings, neurological and endocrine status, surgical outcome, and quality of life in Han Chinese. A retrospective study was conducted on 87 patients treated by trans-sphenoidal surgery, between 2006 and 2011, at a single treatment center in Shandong Provincial Hospital, China. Features of CD were compared and quality of life was analyzed between genders. The female-to-male ratio was 2.78: 1. Results showed that men have a younger age of diagnosis $(\mathrm{P}<0.001)$, a larger adenoma diameter $(\mathrm{P}<0.001)$, and a higher invasion rate $(\mathrm{P}=0.032)$ and apoplexy rate $(\mathrm{P}=0.04)$ than women. To be specific, compared with women, men are more prone to suffering from osteoporosis, hypokalemia, sexual dysfunction, and hypertension ( $\mathrm{P}<0.05$ ), have significantly higher preoperative and postoperative (six months after surgery) cortisol levels $(\mathrm{P}<0.001, \mathrm{P}=0.003)$ and a higher recurrence rate $(30.43 \%$ vs. $7.81 \% ; \mathrm{P}=0.028)$. No significant differences were seen in the CushingQoL scores between genders. Therefore, male patients with $\mathrm{CD}$ need more careful and long-term follow-up than female patients.
\end{abstract}

Keywords: Cushing's disease, gender differences, transsphenoidal surgery, CushingQoL scores

\section{Introduction}

One of the most common causes of endogenous hypercortisolism is Cushing's disease (CD). CD can result in both physical and psychological symptoms. Mass effect, hormone secretion dysfunction, and postoperative complications can severely impact a patient's life [1]. Treatment objectives are to eliminate possible mass effects of tumour, normalize possible hormonal and metabolic disturbances of patients, and to recover normal health-related quality of life (HRQoL) [2]. The CushingQoL questionnaire used was reliable and practicable, specifically designed for Cushing Syndrome (CS) [3].

$\mathrm{CD}$ is different from other types of pituitary adenomas because it is diagnosed more commonly in women than men [4], showing a pronounced female prevalence. However, whether this gender difference in Han Chinese has any relations with the performance and outcome is unknown. The purposes of this study were 1) to evaluate gender-related differences before and after surgery and 2) to

${ }^{*}$ Corresponding author: Zongming Ren, College of Life Science, Shandong Normal University, Ji'nan 250014, P.R. China. Tel.: +86-531-86182515; Fax: +86-531-86182515; E-mail: zmren@sdnu.edu.cn.

0959-2989/14/\$27.50 @ 2014 - IOS Press and the authors. 
use a CushingQoL assessment in a 5-year cohort of surgically treated patients. Therefore, our study focused on the differences between genders pertaining to the clinical symptoms and features, neurological and endocrine status, imaging performance, surgical outcome, and HRQoL in CD patients via a retrospective chart review. We believe that this study will provide innovative insights for this area of research as well as help to ameliorate gender-related diagnosis and treatment of CD.

\section{Materials and methods}

\subsection{Patients}

From 2006 to 2011, 87 patients suffering from an adrenocorticotropic hormone (ACTH)-secreting pituitary adenoma had been managed at the Department of Neurosurgery, Shandong Provincial Hospital in Eastern China, with the male-to-female ratio being 1:2.78 (23 men and 64 women). All patients completed questionnaires during the follow-up. The inclusion criteria for patients are shown in Table 1. Data were collected including age, gender, clinical presentation, past medical history, imaging, neurological and endocrine status, intra-operative findings, and postoperative outcomes and questionnaires. Patients were divided into two groups based on gender. The definition interval was from onset of symptoms to diagnosis. Ethical approval was obtained through the Ethics Committee of Shandong Provincial Hospital. Informed consent was obtained from each individual.

\subsection{Imaging and intra-operative characteristics}

According to Lania et al. [5], the adenomas were subdivided into microadenomas $(<10 \mathrm{~mm})$ and macroadenomas $(>10 \mathrm{~mm})$. The invasiveness of adenomas was assessed according to the preoperative Magnetic Resonance Imaging (MRI) and operative findings. Cavernous sinus invasion (CSI) was diagnosed when the adenoma was seated to the internal carotid artery on MRI or when the cavernous sinus was found by operation [6]. The apoplexy was due to the expansion of a pituitary tumour by infarction, haemorrhage, or hemorrhagic infarction.

Table 1

Inclusion criteria for patients

\begin{tabular}{llll}
\hline & & \multicolumn{1}{c}{ Inclusion criteria } \\
\hline 1 & Classical symptoms & 6 & $\begin{array}{l}\text { Urinary free and/or serum cortisol suppressed by }>50 \% \text { of the baseline value } \\
\text { after oral loading with } 16 \text { mg dexamethasones }\end{array}$ \\
2 & $\begin{array}{l}\text { Increased serum cortisol levels } \\
\text { Loss of diurnal cortisol }\end{array}$ & 7 & Concordant ACTH increase by $50 \%$ of baseline in response to CRH \\
3 & 8 & $\begin{array}{l}\text { Adenoma with positive ACTH immunochemistry } \\
\text { secretion }\end{array}$ \\
& $\begin{array}{l}\text { Increased 24-hour urine-free } \\
\text { cortisol }\end{array}$ & 9 & Presence of a pituitary mass on MRI \\
5 & $\begin{array}{l}\text { Normal/slightly elevated plasma ACTH and/or lack of suppression of urine-free and serum cortisol following oral } \\
\text { Loading with 2 mg dexamethasones }\end{array}$ \\
\hline
\end{tabular}




\subsection{Endocrine assessment}

Morning fasting basal hormone were assessed. Cortisol was measured using an electrochemiluminescent immunoassay kit (Roche Diagnostics GmbH, Mannheim, Germany) with a functional sensitivity of $\leq 8.5 \mathrm{nmol} / \mathrm{L}$. Reference values of the laboratory were $171-536 \mathrm{nmol} / \mathrm{L}$. The intra-assay and inter-assay variation coefficients were $\leq 10 \%$ and $\leq 15 \%$, respectively. Glucocorticoid replacement therapy was stopped three days before every evaluation.

\subsection{Immunohistochemistry}

Pathological evaluation was performed. Tissues were fixed in $10 \%$ formalin, stained using the hematoxylin-eosin method, embedded in paraffin, and examined after immunohistochemical staining with the antibodies against adrenocorticotropic hormone (ACTH), prolactin, growth hormone, follicle-stimulating hormone, luteinising hormone, and thyroid-stimulating hormone.

\subsection{CushingQoL questionnaire}

Patients were requested to complete the questionnaire two years after surgery. The questionnaire followed a standard methodology with a time frame pertaining to the preceding four weeks. It included 12 relevant issues and answers based on five categories: 'Always' 'Often' 'Sometimes' 'Rarely' 'Never', or 'Very much' 'Quite a bit' 'Somewhat' 'Very little' 'Not at all' (scored from 1 to 5) [3]. According to Webb [3], the total score was calculated by adding each issue score (range: 12-60) and transformed from 0 to 100 . Better Quality of Life was also represented by higher scores.

\subsection{Statistical analysis}

Analysis was performed by the SPSS 16.0 (Chicago, IL, USA), while a statistical level of significance of 0.05 was used. The measured quantitative data were presented as mean \pm standard deviation and compared by Mann-Whitney $U$ test. Qualitative data were presented as percentages and compared using a Chi-square test.

\section{Results}

\subsection{Demographic and tumour features}

The clinical features of the patients and adenomas are shown in Table 2 and Table 3 . In total, 87 patients ( $43.33 \pm 10.63$ years old ranging from 23 to 65 years) were studied. For men, the mean age and mean time interval were $30.33 \pm 10.71$ years (range: $23-65$ ) and $55.92 \pm 49.32$ months (range: $0.5-84$ ), respectively; the mean diameter of the adenomas was $1.56 \pm 1.02 \mathrm{~cm}$ (range: $0.6-4.0)$; invasiveness was recognized to be $56.52 \%$ and the apoplexy rate be $17.39 \%$. On the other hand, there were 64 women with a mean age of $48.00 \pm 14.6$ years (range: $23-55$ ) and a mean time interval of $33.09 \pm 34.25$ months (range: 0.1-18). The tumour size was $0.97 \pm 0.55 \mathrm{~cm}$ (range: $0.5-3.2$ ) with an invasion rate and apoplexy rate of $31.25 \%$ and $3.12 \%$, respectively, in female patients. 
Table 2

Demographic and tumour characteristics of patients in the two groups

\begin{tabular}{llll}
\hline Variables & Men $(\mathrm{n}=23)$ & Women $(\mathrm{n}=64)$ & $\mathrm{P}$ \\
\hline Mean age (years) & $30.33 \pm 10.71$ & $48.00 \pm 14.60$ & $<0.001$ \\
Time interval (months) & $55.92 \pm 49.32$ & $33.09 \pm 34.25$ & 0.017 \\
Tumour size (cm) & $1.56 \pm 1.02$ & $0.97 \pm 0.55$ & $<0.001$ \\
\hline
\end{tabular}

Table 3

Dexamethasone suppression test, invasiveness, and apoplexy in the two groups

\begin{tabular}{|c|c|c|c|c|}
\hline Variables & & $\operatorname{Men}(n=23)$ & Women $(n=64)$ & $\mathrm{P}$ \\
\hline \multirow{2}{*}{ Low-dose of dexamethasone } & Suppression & $2(8.70 \%)$ & $4(6.25 \%)$ & 0.653 \\
\hline & Non- suppression & $21(91.30 \%)$ & $60(93.75 \%)$ & \\
\hline \multirow{2}{*}{ High-dose of dexamethasone } & Suppression & $14(60.87 \%)$ & $60(93.75 \%)$ & 0.001 \\
\hline & Non- suppression & $9(39.13 \%)$ & $4(6.25 \%)$ & \\
\hline \multirow{2}{*}{ Invasiveness } & + & $13(56.52 \%)$ & $20(31.25 \%)$ & 0.032 \\
\hline & - & $10(43.48 \%)$ & $44(68.75 \%)$ & \\
\hline \multirow{2}{*}{ Apoplexy } & + & $4(17.39 \%)$ & $2(3.12 \%)$ & 0.040 \\
\hline & - & $19(82.61 \%)$ & $62(96.88 \%)$ & \\
\hline
\end{tabular}

Overall, cases in men were younger than those presenting in women $(\mathrm{P}<0.001)$. Tumour size appeared to be greater in men $(\mathrm{P}<0.001)$. In addition, there were statistically significant differences in mean time interval $(\mathrm{P}=0.017)$, high-dose dexamethasone suppression test $(\mathrm{P}=0.001)$, invasiveness $(\mathrm{P}=0.032)$ and apoplexy $(\mathrm{P}=0.04)$ between the two groups. However, a study of low-dose dexamethasone suppression test did not demonstrate meaningful differences between the two groups.

\subsection{Preoperative symptoms}

Tumour-specific clinical features are presented in Table 4. The most frequent clinical signs encountered in the two groups were central obesity, moon faces, plethora and acne. Additionally, osteoporosis ( $65.22 \%$ vs $35.94 \%$ ), hypokalemia ( $69.57 \%$ vs $43.75 \%)$, sexual dysfunction $(78.26 \%$ vs $37.50 \%)$ and hypertension $(91.3 \%$ vs $65.63 \%)$ occurred more frequently in men compared with women $(\mathrm{P}<0.05)$.

Table 4

Clinical manifestations and symptoms in the two groups

\begin{tabular}{lll}
\hline Variables & Men $(\mathrm{n}=23)$ & Women $(\mathrm{n}=64)$ \\
\hline Central obesity & $18(78.26 \%)$ & $54(84.38 \%)$ \\
Moon face & $18(78.26 \%)$ & $53(82.81 \%)$ \\
Plethora & $17(73.91 \%)$ & $47(73.44 \%)$ \\
Osteoporosis & $15(65.22 \%)^{\mathrm{a}}$ & $23(35.94 \%)^{\mathrm{a}}$ \\
Hypokalemia & $16(69.57 \%)^{\mathrm{a}}$ & $28(43.75 \%)^{\mathrm{a}}$ \\
Acne & $16(69.57 \%)$ & $47(73.44 \%)$ \\
Sexual dysfunction & $18(78.26 \%)^{\mathrm{a}}$ & $24(37.50 \%)^{\mathrm{a}}$ \\
Glucose metabolism dysfunction $^{\mathrm{a}}$ & $2(8.70 \%)$ & $5(7.81 \%)$ \\
Hypertension & $21(91.30 \%)^{\mathrm{a}}$ & $42(65.63 \%)^{\mathrm{a}}$ \\
\hline
\end{tabular}




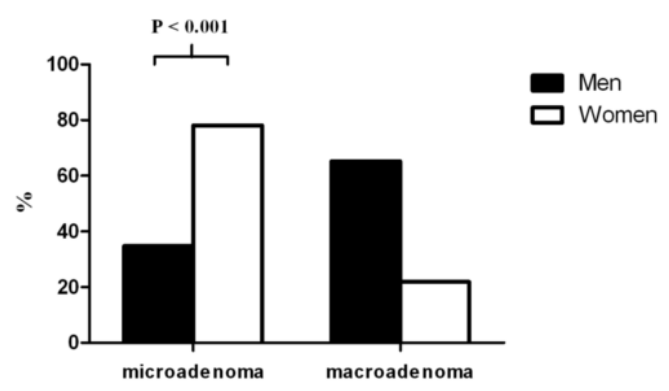

Fig. 1. Classification of tumours in the two groups.

Table 5

Pre- and postoperative cortisol levels in the two groups (unit: nmol/L)

\begin{tabular}{llll}
\hline Pre- and postoperative cortisol levels & Men $(\mathbf{n}=\mathbf{2 3})$ & Women $(\mathbf{n}=\mathbf{6 4})$ & $\mathbf{P}$ \\
\hline Before surgery & $839.88 \pm 308.47$ & $544.77 \pm 200.63$ & $<0.001$ \\
3 days after surgery & $97.15 \pm 72.08$ & $89.54 \pm 89.30$ & 0.714 \\
6 months after surgery & $313.75 \pm 314.49$ & $167.10 \pm 128.48$ & 0.003 \\
\hline
\end{tabular}

\section{3. $M R I$}

Approximately $78.12 \%$ women had microadenomas while $21.88 \%$ had macroadenomas (Figure 1 ). Approximately $34.78 \%$ men had microadenomas while $65.22 \%$ had macroadenomas. Therefore, macroadenomas were mostly associated with men rather than with women $(\mathrm{P}<0.001)$.

\subsection{Hormonal results before and after surgery}

Cortisol levels in men decreased from $839.88 \pm 308.47 \mathrm{nmol} / \mathrm{L}$ before surgery to $97.15 \pm 72.08 \mathrm{nmol} / \mathrm{L}$ in 3 days, and then increased to $313.75 \pm 314.49 \mathrm{nmol} / \mathrm{L}$ in 6 months after surgery (Table 5). For women, cortisol levels decreased from $544.77 \pm 200.63 \mathrm{nmol} / \mathrm{L}$ before surgery to $89.54 \pm 89.30 \mathrm{nmol} / \mathrm{L}$ in 3 days, and then increased to $167.10 \pm 128.48 \mathrm{nmol} / \mathrm{L}$ in 6 months after surgery. One can see that the preoperative cortisol level in men was significantly higher than that in women $(\mathrm{P}<0.001)$, and remains higher in 6 months after surgery $(\mathrm{P}=0.003)$, however, there was no significant difference in cortisol levels in 3 days after surgery between the two groups $(\mathrm{P}=0.714)$.

\subsection{Follow up}

The median duration of follow-up was 45 months (range: 13-121). The recurrence rates for men and women were $30.43 \%$ and $7.81 \%(\mathrm{P}=0.013)$, respectively. Based on postoperative hormone levels at 6 months, cortisol was controlled in $78.26 \%$ of men and $95.31 \%$ of women. The cortisol normalization rate in men was also significantly lower compared with women $(\mathrm{P}=0.028)$. 
Table 6

CushingQoL scores in the two groups

\begin{tabular}{llll}
\hline CushingQoL scores & Men(n= 23) & Women(n= 64) & P \\
\hline Somnipathy & $3.43 \pm 0.84$ & $2.94 \pm 1.08$ & 0.049 \\
Have pain from leading a normal life & $3.39 \pm 1.03$ & $3.78 \pm 1.09$ & 0.140 \\
Take a long time to heal wounds & $2.91 \pm 1.00$ & $3.47 \pm 1.04$ & 0.029 \\
Bruise easily & $3.00 \pm 0.90$ & $3.89 \pm 0.91$ & $<0.001$ \\
More irritable & $3.70 \pm 1.15$ & $3.09 \pm 0.94$ & 0.015 \\
Less self-confidence and more insecure & $2.83 \pm 1.07$ & $2.98 \pm 1.06$ & 0.542 \\
Worried about physical changes due to illness & $2.96 \pm 0.88$ & $2.61 \pm 0.77$ & 0.077 \\
Less like going out & $3.26 \pm 0.92$ & $2.64 \pm 0.74$ & 0.002 \\
Had to give up social activities & $3.17 \pm 1.03$ & $3.20 \pm 0.84$ & 0.893 \\
Illness affects everyday activities & $3.43 \pm 1.08$ & $3.56 \pm 0.92$ & 0.588 \\
Difficult to remember things & $3.91 \pm 1.00$ & $4.05 \pm 0.84$ & 0.536 \\
Worried about health in the future & $2.87 \pm 1.10$ & $3.14 \pm 0.83$ & 0.224 \\
\hline
\end{tabular}

\subsection{CushingQoL scores}

CushingQoL scores in men and women were 55.98 \pm 17.88 and $57.00 \pm 15.32$, respectively. No statistically significant difference was observed in the CushingQoL scores (Table 6).

\section{Discussion}

The research in this study depicted a pattern for Chinese Han patients with CD with men generally being diagnosed with greater tumours than women although the majority of patients were diagnosed in their thirties and forties, men appeared to be younger than women. These findings were consistent with those reported in the literature [7]. Furthermore, macroadenomas were mostly associated with men rather than with women, which might be due to the facts that a delayed diagnosis may allow tumours to grow larger [8], which men tend to feel uncomfortable complaining about their sexual problems, or that women usually present evident symptoms such as menstrual disorders. Caputo [9] and Nabarro [10] reported that premenopausal women may have smaller adenomas caused by menstrual abnormalities, while others showed that the rate of invasiveness and apoplexy were higher in men than in women. Further, in accordance with Cukiert [11], macroadenomas may be also related to cavernous sinus invasion. In the pituitary fossa, the absence of a bony interface could explain the invasion.

Our study showed that male patients tend to have a more severe clinical presentation than women. Chronic exposure to glucocorticoid may result in moon face, central obesity, hypertension, osteoporosis, fatigue, insomnia, depression, anxiety, cognitive problems [12]. Such manifestations (osteoporosis and psychosis of excessive cortisol production) were also more frequent in men than in women [13]. Some authors reported parallel data concerning the prevalence of osteoporosis in men, whereas others disagreed [14,15]. Feelders [1] identified hypertension and osteoporosis as significant risk factors in CD patients and revealed that a decrease in osteoblastic activity and an increase in osteoclastic bone resorption may result in osteoporosis. Additionally, our study suggested that males were found to be an independent risk factor for osteoporosis. We agree that hypertension also contributes to the markedly increased mortality of increased cardiovascular risk and that women are more likely to seek early medical advice because of the presentation of severe symptoms, such as 
menstrual irregularities or changed appearance. Our results were also in accordance with those described by Vierhapper [4] that the sex-specific difference is of clinical relevance in patients with hypercortisolism. Finally, concerning the hormone levels, both preoperative cortisol and postoperative cortisol in men were higher than those in women. Furthermore, their postoperative hormone levels in six months showed that the cortisol normalization rate was significantly lower in men than in women. This is in agreement with previous studies [16] that adenomas secreting ACTH occurred more frequently in women.

At present, contemporary treatment options of $\mathrm{CD}$ are limited and may pose additional risks. In our study, the recurrence rate in patients, $13.79 \%$, was lower than that reported by others [17] with recurrence rates for males $30.43 \%$ and females $7.81 \%$. Our results also indicated that men not only have greater but also have more invasive tumours with a possibility of lower recurrence rate reflecting long-term remission. We feel that the elevated rate of recurrence in male patients is an important finding, suggesting that careful long-term follow-up be needed in this subset of patients even if surgery is considered successful.

Finally, reduced HRQoL is a common complaint of patients suffering from pituitary tumours. HRQoL assessment gives clinician important information on patients' everyday life. Recently, the CushingQoL questionnaire was deemed reliable and valid for Cushing's syndrome [3]. From the results of our study, CushingQoL scores in male and female patients were 55.98 \pm 17.88 and $57.00 \pm 15.32$, respectively, which were similar to those reported in recent research [18]. Furthermore, there was no significant difference in the scores between genders. However, male patients scored lower on physical symptoms such as tendency to bruise and taking a long time to heal wounds than females, while females scored lower on the somnipathy and psychiatric symptoms (such as becoming more irritable, less like going out). These results were in accordance with those reported by Pecori et al. [7] that somnipathy and psychiatric symptoms were more common and more easily impaired in women than in men. Hirsch [13] also reported that patients often feel fatigue, emotional instability, depression, and sleeping difficulties.

Previously reported data appear to corroborate the findings that CD is more common in women than in men [19]. However, despite the abundance of published data concerning various aspects of the clinical course and treatment of $\mathrm{CD}$, few investigations have paid attention to the clinical features, laboratory findings and HRQoL assessment between genders. In an attempt to distinguish such differences, our study investigated 87 patients who underwent surgery for a period of five years to conclude a gender-dependent difference in presentation and postoperative outcomes in CD patients. Our findings suggested that longer-term follow-up, increased awareness and earlier diagnosis in men could be helpful in improving the treatment of CD. It is our hope that our research will provide innovative insights in this important field.

\section{Acknowledgment}

This study was financially supported by the national natural science foundation of China (21107135).

\section{References}

[1] R.A. Feelders, S.J. Pulgar, A. Kempel and A.M. Pereira, The burden of Cushing's disease: clinical and health-related quality of life aspects, European Federation of Endocrine Societies 167 (2012), 311-326. 
[2] M.O. van Aken, A.M. Pereira, N.R. Biermasz, S.W. van Thiel and J.W. Smit, Quality of life in patients after long-term biochemical cure of Cushing's disease, The Journal of Clinical Endocrinology and Metabolism 90 (2005), 3279-3286.

[3] S.M. Webb, X. Badia, M.J. Barahona, A. Colao, C.J. Strasburger and A. Tabarin, Evaluation of health-related quality of life in patients with Cushing's syndrome with a new questionnaire, European Journal of Endocrinology/European Federation of Endocrine Societies 158 (2008), 623-630.

[4] H. Vierhapper, P. Nowotny and W.Waldhausl, Sex-specific differences in cortisol production rates in humans, Metabolism: Clinical and Experimental 47 (1998), 974-976.

[5] A. Lania and P. Beck-Peccoz, Pituitary incidentalomas, Best Practice \& Research Clinical Endocrinology \& Metabolism 26 (2012), 395-403.

[6] E. Knosp, K. Kitz, E. Steiner and C. Matula, Pituitary adenomas with parasellar invasion, Acta Neurochirurgica Supplementum 53 (1991), 65-71.

[7] F. Pecori Giraldi, M. Moro and F. Cavagnini, Gender-related differences in the presentation and course of Cushing's disease, The Journal of Clinical Endocrinology and Metabolism 88 (2003), 1554-1558.

[8] B.D. McDowell, R.B. Wallace, R.M. Carnahan, E.A. Chrischilles and C.F. Lynch, Demographic differences in incidence for pituitary adenoma, Pituitary 14 (2011), 23-30.

[9] C. Caputo, T. Sutherland, S. Farish and P. McNeill, Gender differences in presentation and outcome of nonfunctioning pituitary macroadenomas, McNeill, Clinical Endocrinology 78 (2013), 564-570.

[10] J.D. Nabarro, Pituitary prolactinomas, Clinical Endocrinology 17 (1982), 129-155.

[11] A. Cukiert, M. Andrioli, J. Goldman, Salgado and M. Knoepfelmacher, Cavernous sinus invasion by pituitary macroadenomas, Neuroradiological, Clinical and Surgical Correlation, Arquivos de Neuro-Psiquiatria 56 (1998), $107-110$.

[12] A. Santos, E. Resmini, I. Crespo, E. Valassi and M. Roset, Psychometric performance of the CushingQoL questionnaire in conditions of real clinical practice, European Journal of Endocrinology 167 (2012), 337-342.

[13] D. Hirsch, G. Orr, V. Kantarovich, H. Hermesh, E. Stern and I. Blum, Cushing's syndrome presenting as a schizophrenia-like psychotic state, The Israel Journal of Psychiatry and Related Sciences 37 (2000), 46-50.

[14] R.C. Urbanic and J.M. George, Cushing's disease-18 years' experience, Medicine 60 (1981), 14-24.

[15] E.J. Ross and D.C. Linch, Cushing's syndrome-killing disease: discriminatory value of signs and symptoms aiding early diagnosis, Lancet 2 (1982), 646-649.

[16] K.Y. Ho, W.S. Evans, R.M. Blizzard, J.D. Veldhuis, G.R. Merriam and E. Samojlik, Effects of sex and age on the 24-hour profile of growth hormone secretion in man: importance of endogenous estradiol concentrations, The Journal of Clinical Endocrinology and Metabolism 64 (1987), 51-58.

[17] C. Dimopoulou, J. Schopohl, W. Rachinger, M. Buchfelder, J. Honegger, M. Reincke and G.K. Stalla, Long-term remission and recurrence rates after first and second transsphenoidal surgery for Cushing's disease: care reality in the Munich Metropolitan Region, Reincke, European Journal of Endocrinology/European Federation of Endocrine Societies 170 (2014), 285-294.

[18] J. Tiemensma, A.A. Kaptein, A.M. Pereira, J.W. Smit, J.A. Romijn and N.R. Biermasz, Negative illness perceptions are associated with impaired quality of life in patients after long-term remission of Cushing's syndrome, European Journal of Endocrinology / European Federation of Endocrine Societies 165 (2011), 527-535.

[19] E.L. Mazzaferri and R.T. Kloos, Clinical review 128: Current approaches to primary therapy for papillary and follicular thyroid cancer, The Journal of Clinical Endocrinology and Metabolism 86 (2001), 1447-1463. 